\title{
Stabilization of submillimeter dimensions: The new guise of the hierarchy problem
}

\author{
Nima Arkani-Hamed \\ SLAC, Stanford University, Stanford, California 94309 \\ Savas Dimopoulos \\ Physics Department, Stanford University, Stanford, California 94305 \\ John March-Russell \\ Theory Division, CERN, CH-1211, Geneva 23, Switzerland
}

(Received 9 February 1999; published 20 February 2001)

\begin{abstract}
A new framework for solving the hierarchy problem was recently proposed which does not rely on low energy supersymmetry or technicolor. The fundamental Planck mass is at TeV and the observed weakness of gravity at long distances is due to the existence of new submillimeter spatial dimensions. In this picture the standard model fields are localized to a $(3+1)$-dimensional wall or "3-brane." The hierarchy problem becomes isomorphic to the problem of the largeness of the extra dimensions. This is in turn inextricably linked to the cosmological constant problem, suggesting the possibility of a common solution. The radii of the extra dimensions must be prevented from both expanding to too great a size, and collapsing to the fundamental Planck length $\mathrm{TeV}^{-1}$. In this paper we propose a number of mechanisms addressing this question. We argue that a positive bulk cosmological constant $\bar{\Lambda}$ can stabilize the internal manifold against expansion, and that the value of $\bar{\Lambda}$ is not unstable to radiative corrections provided that the supersymmetries of string theory are broken by dynamics on our 3-brane. We further argue that the extra dimensions can be stabilized against collapse in a phenomenologically successful way by either of two methods: (1) large, topologically conserved quantum numbers associated with higher-form bulk U(1) gauge fields, such as the naturally occurring Ramond-Ramond gauge fields, or the winding number of bulk scalar fields; (2) the brane-lattice crystallization of a large number of 3-branes in the bulk. These mechanisms are consistent with theoretical, laboratory, and cosmological considerations such as the absence of large time variations in Newton's constant during and after primordial nucleosynthesis, and millimeter-scale tests of gravity.
\end{abstract}

DOI: 10.1103/PhysRevD.63.064020

PACS number(s): 04.50.+h

\section{NEW GUISE OF THE HIERARCHY PROBLEM}

A new proposal for solving the hierarchy problem was recently introduced [1-3] which circumvents the need for supersymmetry or technicolor. Instead the hierarchy problem for the standard model (SM) is solved by bringing the fundamental Planck scale down to the TeV scale. Gravity becomes comparable in strength to the other interactions at this scale, and the observed weakness of gravity at long distances is then explained by the presence of $n$ new "large" spatial dimensions.

Gauss' Law relates the Planck scales of the $(4+n)$-dimensional theory, $M_{*}$, and the long-distance 4-dimensional theory, $M_{\mathrm{pl}}$,

$$
M_{\mathrm{pl}}^{2} \sim r_{n}^{n} M_{*}^{n+2}
$$

where $r_{n}$ is the size of the extra dimensions. Putting $M_{*}$ $\sim 1 \mathrm{TeV}$ then yields

$$
r_{n} \sim 10^{-17+(30 / n)} \mathrm{cm}
$$

For $n=1, r_{1} \sim 10^{13} \mathrm{~cm}$, so this case is excluded since it would modify Newtonian gravity at solar-system distances. Already for $n=2$, however, $r_{2} \sim 1 \mathrm{~mm}$, which happens to be the distance where our present experimental knowledge of gravitational strength forces ends. For larger $n, 1 / r_{n}$ slowly approaches the fundamental Planck scale $M_{*}$.

While the gravitational force has not been measured beneath a millimeter, the success of the SM up to $\sim 100 \mathrm{GeV}$ implies that the SM fields cannot feel these extra large dimensions; that is, they must be stuck on a 3-dimensional wall, or "3-brane,' in the higher dimensional space. Thus, in this framework the universe is $(4+n)$-dimensional with fundamental Planck scale near the weak scale, with $n \geqslant 2$ new submillimeter sized dimensions where gravity, and perhaps other fields, can freely propagate, but where the SM particles are localized on a 3-brane in the higher-dimensional space. The most attractive possibility for localizing the SM fields to the brane is to employ the D-branes that naturally occur in type I or type II string theory [4,2]. Gauge and other degrees of freedom are naturally confined to such D-branes [4], and furthermore this approach has the obvious advantage of being formulated within a consistent theory of gravity. However, from a practical point of view, the most important question is whether this framework is experimentally excluded. This was the subject of [3] where laboratory, astrophysical, and cosmological constraints were studied and found not to exclude these ideas.

There are also a number of other important papers discussing related suggestions. References [5] examine the idea of lowering the grand unified theory (GUT) scale by utilizing higher dimensions. Further papers concern themselves with 
the construction of string models with extra dimensions larger than the string scale [6-8], and gauge coupling unification in higher dimensions without lowering the unification scale [9]. There are also two important papers by Sundrum. The first deals with the effective theory of the low energy degrees of freedom in realizations of our world as a brane [10], while the second is concerned with the topic of radius stabilization [11], and with which our analysis has much in common.

In our framework the hierarchy problem becomes the problem of explaining the size and stability of the large extra dimensions. The main purpose of this paper is to exhibit mechanisms which accomplish these objectives, and examine some aspects of their phenomenology. Since a rather wide collection of possible stabilization mechanisms are discussed in this paper, only some of which we believe to be successful, we think it useful to provide the reader with a guide to our main results: In Sec. I A we discuss a very general consistency constraint on the bulk cosmological constant; and in Sec. II we describe some basic kinematics pertaining to the radial oscillation field, whose mass will turn out to provide significant constraints on stabilization scenarios. In particular this is the constraint that will sometimes force us to have a large conserved integer parameter in our models. In Sec. III we show that the properties and limits on such light radial oscillation fields can be discussed in a way that is independent of the details of the precise radiusstabilization mechanism. We also briefly describe the reasons for the cosmological safety of this scenario. The most important results of this paper are contained in Sec. IV where we discuss long-distance (IR) and, particularly, shortdistance (UV) stabilization mechanisms, and put these together to obtain a variety of complete stabilization models. We find that two methods of UV stabilization are particularly attractive: "brane-lattice-crystallization" discussed in Sec. IV B; and "topological stabilization"' discussed in Sec. IV C. Finally in Sec. V we present a summary of our results.

\section{A. The hierarchy and the bulk cosmological constant}

Let us begin with some necessary conditions that must be satisfied to ensure the existence of large radii. As we know from experience with our 4-dimensional world, to ensure that our three ordinary spatial dimensions are very large the radius of curvature of the universe must be no less than the present horizon size. This leads to the requirement that the cosmological constant of the universe is less than the critical density. An identical line of reasoning for the case of $n$-extra dimensions also leads to an upper limit on the bulk cosmological constant as we now explain [12].

The curvature radius $L_{\text {curv }}$ of the bulk space in the presence of energy density or an effective cosmological constant, $\bar{\Lambda}$, in the bulk, is

$$
L_{\text {curv }} \sim\left(\frac{M_{*}^{n+2}}{\bar{\Lambda}}\right)^{1 / 2}
$$

This curvature radius must be larger than the physical size of the transverse dimensions $r_{n}$ in order to ensure that the bulk space does not "split off" into separate inflating universes separated by horizons of size $L_{\text {curv }}$, or collapse into black holes. This gives an upper bound on $\bar{\Lambda}[12]$ :

$$
\bar{\Lambda} \lesssim M_{*}^{(4+n)}\left(\frac{M_{*}}{M_{\mathrm{pl}}}\right)^{4 / n} .
$$

This constraint will play an important role in what follows. It already implies that the magnitude $\bar{\Lambda}$ must be smaller than the fundamental scale of $M_{*}$. This was to be expected since in this case there is one scale in the problem and the bulk would split into a collection of non-communicating $1 /$ tev size regions, outside of each others' particle horizons. An important corollary of this is that one cannot use the ScherkSchwarz mechanism to break supersymmetry at $M_{*}$ since this would induce a bulk cosmological constant of the order of $M_{*}^{4+n}$, which exceeds the limit Eq. (4).

Of course the effective 4-dimensional cosmological constant measured at long distances (greater than the size of the extra dimensions) must to a very high degree of accuracy vanish. This can be achieved by canceling the wall and bulk contributions against one another: ${ }^{1}$

$$
0=f^{4}+\left(r_{n}\right)^{n} \bar{\Lambda} .
$$

We see that if the bulk energy is negative, a positive $f^{4}$ will cancel the 4-dimensional cosmological constant, while if the bulk energy is positive, we need a negative $f^{4}$. Clearly a positive $f^{4}$ is reasonable; if the wall can fluctuate in the extra dimensions, $f^{4}$ is just the tension of the wall, and provides the correct sign kinetic term for the Nambu-Goldstone bosons of spontaneously broken $(4+n)$-dimensional Poincaré invariance which live on the wall. This reasoning seems to exclude the possibility of a negative $f^{4}$, since this gives the wrong sign kinetic term to the Nambu-Goldstone bosons. This is however only a problem if the Nambu-Goldstone fields are indeed present, that is, if the $(4+n)$-dimensional Poincare invariance is spontaneously broken. On the other hand, suppose that the wall is "stuck" and cannot fluctuate in the extra dimensions, due to explicit breaking of $(4+n)$-dimensional Poincaré invariance. As an example, we can consider twisted sector fields living at an orbifold fixed point. In the language of string theory the wall could be an orientifold rather than a D-brane. In this case, $f^{4}$ is just the wall energy density acting as a source for gravity, but there are no Nambu-Goldstone bosons on the wall to receive a wrong-sign kinetic term. Another way of saying this is as follows. The wall can have an energy density as a source for gravity $f_{\text {grav }}^{4}$, and a tension under "bending" $f_{\text {bend }}^{4}$. It is $f_{\text {grav }}^{4}$

\footnotetext{
${ }^{1}$ Note that what we are refering to as " $\Lambda r_{n}^{n}$ ", is simply the bulk energy projected onto 4 dimensions, and receives contributions from all forms of bulk energy and not only the higher dimensional cosmological constant. For instance, for two large dimensions compactified on a sphere, the integral of the internal Ricci scalar over the internal dimensional dimensions yields a negative constant value for $\Lambda r_{n}^{n}$.
} 
which should appear in Eq. (5). If the $(4+n)$-dimensional Poincaré invariance is only spontaneously broken, its nonlinear realization forces $f_{\text {grav }}^{4}=f_{\text {bend }}^{4}$, as they both come from expanding the term in the action

$$
-\int d^{4} \sqrt{-g_{\text {ind }}} f^{4}
$$

where $g_{\text {ind }}$ is the induced metric on the wall. Since $f_{\text {bend }}^{4}$ $>0$, we have $f_{\text {grav }}^{4}>0$. On the other hand, if the $(4+n)$-dimensional Poincaré invariance is explicitly broken, there need not be any relationship between the two. Indeed, if the wall cannot fluctuate, effectively $f_{\text {bend }}^{4}=\infty$, while $f_{\text {grav }}^{4}$ can be finite and of any sign.

We will therefore allow the possibility that a brane can make a net negative contribution to the 4-dimensional cosmological constant, which provides us with the freedom to consider stabilization mechanisms that give either positive or negative bulk energy densities. ${ }^{2}$

Given Eq. (4) we learn that if our wall is the only brane, then its effective wall-localized cosmological constant, $f^{4}$, is bounded above by

$$
f \leqslant M_{*}\left(\frac{M_{\mathrm{pl}}}{M_{*}}\right)^{(n-2) / 2 n} .
$$

This is not too severe a constraint though, varying between $10 \mathrm{TeV}$ for $n=2$, to $\sim 10^{8} \mathrm{GeV}$ for $n=6$. Of course, the relation, Eq. (5), can be turned around to determine the effective bulk cosmological constant, $\bar{\Lambda}$, given $f$. A natural assumption for the wall-localized cosmological constant, given our state of knowledge of the standard model interactions on the wall, is $f^{4}=(1 \mathrm{TeV})^{4} \sim M_{*}^{4}$. Thus in this case

$$
\bar{\Lambda}=M_{*}^{4+n}\left(\frac{M_{*}}{M_{\mathrm{pl}}}\right)^{2}
$$

is the value of the bulk cosmological constant necessary to cancel the total long-distance cosmological constant in our world. Note that this value is indeed always less than the upper bound Eq. (4) arising from the bulk curvature constraint.

Later in Sec. IV B we will consider stabilization mechanisms that utilize many branes populating the bulk. In this case the bounds Eqs. (4) and (7) are modified by the total brane number $N_{\text {wall }}$.

A difference with the previous case is that the curvature radius must now only be greater than the inter-brane separation $r_{n} /\left(N_{\text {wall }}\right)^{1 / n}$. (We are assuming the best case situation of equally spaced branes which leads to the weakest bound.) The reason for this is that the branes themselves are localized sources of curvature of the opposite sign, so that at long distances compared to the inter-brane separation, the curvature of the bulk averages out to zero. From this follows the generalized curvature constraint

\footnotetext{
${ }^{2}$ We thank Eva Silverstein for discussions about this point.
}

$$
\bar{\Lambda} \lesssim N_{\text {wall }} M_{*}^{(4+n)}\left(\frac{M_{*}}{M_{\mathrm{pl}}}\right)^{4 / n}
$$

The IR cancellation of the effective cosmological constant in 4-dimensions is expressed by

$$
0=N_{\text {wall }} 4^{4}+\left(r_{n}\right)^{n} \bar{\Lambda} .
$$

Imposing this leads to the following bound on the walllocalized cosmological constant

$$
f^{4} \lesssim M_{*}^{4} \frac{1}{N_{\text {wall }}^{(n-2) / n}}\left(\frac{M_{\mathrm{pl}}}{M_{*}}\right)^{(2 n-4) / n} .
$$

The cosmological constant is bounded from below from another consideration. As we will see later in Sec. III, there are light gravitationally coupled particles in the spectrum whose (mass) $)^{2}$ is proportional to $\bar{\Lambda}$ [see Eq. (36)]. The requirement that these particles do not conflict with measurements of gravity imply that they weigh more than a $\mathrm{meV}$ and consequently put a lower limit on $\bar{\Lambda}$. This in turn implies that the large size of the new dimensions in most, but not all cases studied here, cannot be solely due to the smallness of $\bar{\Lambda}$. Additional dynamics to boost the size of the extra dimensions are necessary. This can easily come about if there is a conserved charge in the system, analogous to baryon number. Just as humans are large because they carry large baryon number, the extra dimensions can be large because they carry some large charge $Q$. In some of our examples, this charge corresponds to a large number of walls $Q \sim N_{\text {wall }} \gg 1$. In others, it is a topological charge $k$. Note however that in some special cases, it is not necessary to use a large conserved charge. For example, as we discuss in Sec. IV C if the fundamental scale $M_{*}$ is pushed to $\sim 10 \mathrm{TeV}$ while the wall contribution to the cosmological constant $f^{4}$ is kept at $\sim(1 \mathrm{TeV})^{4}$, then topological charges $k \sim 1$ are adequate. This is not too unnatural a situation, especially considering that a loop factor could easily supply such a suppression to $f^{4}$.

\section{B. Stable and calculable hierarchy}

In this paper we will not search for dynamical mechanisms where the hierarchy between the size of the extra dimensions and the fundamental scale is calculable. We will instead be content to enforce this hierarchy by choosing the bulk cosmological constant to be small and/or the abovementioned topological or other charge to be large. This is analogous to the early days of the minimal supersymmetric standard model (MSSM) [13] where the soft supersymmetry breaking terms were postulated without any reference to a dynamical mechanism which generates them. The idea there was that since the problem of supersymmetry breaking is connected with the cosmological constant problem it seemed premature to adopt a specific supersymmetry- (SUSY-) breaking mechanism and it seemed more prudent to study consequences that were independent of the details of the SUSY-breaking mechanics. Similarly, in our new framework 
the hierarchy and cosmological constant problems are even more closely intertwined so we will adopt a similar philosophy of not insisting on a detailed dynamical mechanism for a calculable hierarchy and will be content to instead parametrize our ignorance by a choice of $\bar{\Lambda}$ and an integer $Q\left(N_{\text {wall }}\right.$ or $k$ ).

The second aspect of the hierarchy problem is its stability against radiative corrections. In the MSSM this is guaranteed by low energy supersymmetry, which protects the Higgs mass against large radiative corrections. Presumably, the analogous question in our framework is the behavior of the pair of parameters $(\bar{\Lambda}, Q)$ under radiative corrections. The integer $Q$ is automatically protected since it refers to charge of a configuration. Since $\bar{\Lambda}$ is a bulk cosmological constant one can imagine two possibilities. One is that whatever solves the cosmological constant problem will also prevent $\bar{\Lambda}$ from becoming as large as the cutoff $M_{*}$. The second more explicit and perhaps more satisfactory viewpoint is to invoke bulk-supersymmetry to protect $\bar{\Lambda}$ from large radiative corrections. Indeed, as pointed out in Ref. [2], if supersymmetry is broken solely on our 3-brane by an amount $\sim M_{*}$ $\sim 1 \mathrm{TeV}$, the Fermi-Bose splittings that this induces in the bulk are miniscule $\sim \mathrm{TeV}^{2} / M_{\mathrm{pl}} \sim 10^{-3} \mathrm{eV}$ and therefore the bulk cosmological constant $\bar{\Lambda}$ is protected by the approximate bulk-supersymmetry.

It should be emphasized that stabilizing large dimensions is inherently easier than stabilizing Planck-scale dimensions. In the latter case, quantum gravitational effects are necessarily important and cannot be ignored. However, precisely because we are interested in large radii, the details of short distance physics are largely irrelevant and a classical or semi-classical analysis suffices. We will consider this point more explicitly in Sec. IV.

\section{KINEMATICS OF RADIUS STABILIZATION}

Suppose that we have an $N$-brane embedded in a space with $N$ large spatial dimensions and $n$ small dimensions we wish to stabilize. The total action is comprised of a bulk part,

$$
\begin{aligned}
S_{\text {bulk }}= & -\int d^{1+N+n} x \sqrt{-\operatorname{det} G_{(1+N+n)}}\left(M_{*}^{(n+N-1)} \mathcal{R}+\Lambda\right. \\
& \left.-\mathcal{L}_{\text {matter }}+\ldots\right),
\end{aligned}
$$

and a brane part,

$$
S_{\text {brane }}=-\int d^{1+N} x \sqrt{-\operatorname{det} g_{(1+N)}^{\text {induced }}}\left(f^{N+1}+\ldots\right),
$$

where $\mathcal{L}_{\text {matter }}$ is the Lagrangian of bulk gauge or scalar fields, and the ellipses denote higher-derivative terms that can be ignored in the regime of interest as we will demonstrate below. Take the background metric for the $(1+N+n)$-dimensional spacetime to be of the form

$$
g_{\mu \nu}=\left(\begin{array}{lll}
1 & & \\
& -R(t)^{2} g_{I J} & \\
& & -r(t)^{2} g_{i j}
\end{array}\right),
$$

where $R$ is the scale factor of the $N$-dimensional space, and $r$ is the scale factor of the internal $n$-dimensional space, with geometry set by $g_{i j}$ where $\operatorname{det}\left(g_{i j}\right)=1$.

With this metric the Ricci scalar is

$$
\begin{aligned}
-\mathcal{R}= & 2 N \frac{\ddot{R}}{R}+N(N-1)\left(\frac{\dot{R}}{R}\right)^{2}+2 n \frac{\ddot{r}}{r}+n(n-1)\left(\frac{\dot{r}}{r}\right)^{2} \\
& +2 N n\left(\frac{\dot{r} \dot{R}}{r R}\right)+\frac{\kappa n(n-1)}{r^{2}},
\end{aligned}
$$

where the internal curvature term is present for $n$-spheres $(\kappa=1)$, but vanishes for tori $(\kappa=0)$, and we have ignored a similar curvature term for the large dimensions. After integrating over all spatial coordinates we obtain

$$
S=\int d t\left[\mathcal{L}_{\mathrm{KE}}(\dot{R}, \dot{r})-R^{N} V_{\mathrm{tot}}(r)\right],
$$

where the total potential is given by

$$
\begin{gathered}
V_{\text {tot }}(r)=V_{\text {bulk }}+V_{\text {wall }} \\
V_{\text {wall }}=f^{N+1} \\
V_{\text {bulk }}=\Lambda r^{n}-n(n-1) \kappa M_{*}^{n+N-1} r^{n-2}+V_{\text {matter }}(r)
\end{gathered}
$$

where

$$
V_{\text {matter }}(r)=-\int d^{n} x\left(r^{n} \mathcal{L}_{\text {matter }}\right) .
$$

After integrating the $\ddot{R}$ and $\ddot{r}$ terms by parts, the kinetic part of the action for the radii, $R$ and $r$, becomes

$$
\begin{aligned}
S= & -M_{*}^{N+n-1} \int d t R^{N} r^{n}\left[N(N-1)\left(\frac{\dot{R}}{R}\right)^{2}+n(n-1)\left(\frac{\dot{r}}{r}\right)^{2}\right. \\
& \left.+2 N n\left(\frac{\dot{r} \dot{R}}{r R}\right)\right] .
\end{aligned}
$$

Note the overall negative sign of these kinetic terms. This is connected to the well-known phenomenon that the conformal mode of gravity has the opposite sign kinetic term to the transverse graviton kinetic term (and which bedevils attempts at defining quantum gravity via the Euclidean functional integral).

In any case there is clearly an extremum of the action with $\dot{R}=\dot{r}=0$, when the condition $\left.\partial_{R}\left[R^{N} V_{\text {tot }}(r)\right]\right|_{R=R_{0}, r=r_{0}}$ $=0$, (and similar with $\partial_{R} \rightarrow \partial_{r}$ ) is met. These imply (for $R_{0}$ $\neq 0)$

$$
\begin{gathered}
V_{\text {tot }}\left(r_{0}\right)=0, \quad \text { and } \\
V_{\text {tot }}^{\prime}\left(r_{0}\right)=0 .
\end{gathered}
$$


This is as one would have naively expected. However, because of the negative sign for the kinetic term for the radial degrees of freedom, the stability analysis for such static solutions has to be treated with care. The analysis starts by expanding the action, Eq. (19), in small fluctuations around the extremum: $R(t)=R_{0}+\delta R(t)$, and $r(t)=r_{0}+\delta r(t)$. Then to quadratic order, and defining $\Delta \equiv \delta R / R_{0}$ and $\delta$ $\equiv \delta r / r_{0}$, the expansion gives the coupled equations of motion

$$
\left(\begin{array}{cc}
N(N-1) & N n \\
N n & n(n-1)
\end{array}\right)\left(\begin{array}{c}
\ddot{\Delta} \\
\ddot{\delta}
\end{array}\right)=\left(\begin{array}{cc}
0 & 0 \\
0 & \omega^{2}
\end{array}\right)\left(\begin{array}{c}
\Delta \\
\delta
\end{array}\right),
$$

where

$$
\omega^{2}=\frac{1}{2} \frac{\left(r_{0}\right)^{2} V_{\mathrm{tot}}^{\prime \prime}\left(r_{0}\right)}{M_{*}^{N+n-1}\left(r_{0}\right)^{n}}=\frac{1}{2} \frac{\left(r_{0}\right)^{2} V_{\mathrm{tot}}^{\prime \prime}\left(r_{0}\right)}{M_{(N+1)}^{N-1}}
$$

Here $M_{(N+1)}$ is to be understood as the effective Planck mass in the large $(N+1)$-dimensional spacetime $\left(M_{(4)}\right.$ $\left.\equiv M_{\mathrm{pl}}\right)$. We now search for oscillating solutions, $(\Delta, \delta)$ $=\exp (i \Omega t)\left(\Delta_{0}, \delta_{0}\right)$ of the stability equations. From Eq. (21), $\Omega^{2}$ is thus given by the eigenvalues of the matrix

$$
+\frac{\omega^{2}}{n N(N+n-1)}\left(\begin{array}{cc}
0 & -N n \\
0 & N(N-1)
\end{array}\right),
$$

namely, $\Omega^{2}=0$, and

$$
\Omega^{2}=\frac{N-1}{n(N+n-1)} \omega^{2}
$$

The zero eigenvalue just corresponds to the fact that $R_{0}$ is a flat direction since, by assumption, there is no potential for $R$. The crucial expression is Eq. (24), which gives us the condition for stability of our static solution. Stability requires $\Omega^{2}>0$, which for $N>1$ implies

$$
\omega^{2}>0 \Rightarrow V_{\text {tot }}^{\prime \prime}\left(r_{0}\right)>0 \text {. }
$$

This is the main result of this section. Even though it seems trivial that stability is equivalent to requiring the second derivative of the potential around the extremum to be positive, this condition is a priori not at all obvious given the negative kinetic terms for the radii fields. As an example of this consider the case $N=0$, which corresponds to $r$ being thought of as the radius of a Friedman-Robertson-Walker universe. In this case stability requires $\omega^{2}<0$, or equivalently $V_{\mathrm{tot}}^{\prime \prime}\left(r_{0}\right)$ $<0$. This accords with our usual understanding: for example take the only term in $V$ to be a positive cosmological term $V_{\text {tot }}(r)=\Lambda r^{n}$. Then around the minimum at $r=0$ the solution is unstable to inflationary growth as we expect.

The end result of this analysis is simply that we can think in terms of a total potential $V(r)$ that one can minimize to find the stable static solutions for the size of the internal dimensions. Also note that from Eqs. (24) and (22) we can extract the mass of the canonically normalized radial oscillation field $\phi$ (we will refer to $\phi$ as the "radion") in the case of interest, $N=3, n$ arbitrary:

$$
m_{\text {radial }}^{2}=\frac{1}{n(n+2)} \frac{\left(r_{0}\right)^{2} V_{\mathrm{tot}}^{\prime \prime}\left(r_{0}\right)}{M_{\mathrm{pl}}^{2}} .
$$

Notice that as a consequence, the magnitude of $\phi$ is related to the deviation $\delta r$ from the equilibrium radius $r=r_{0}+\delta r$ via

$$
\frac{\delta r}{r_{0}} \sim \frac{\phi}{M_{\mathrm{pl}}}
$$

\section{MODEL-INDEPENDENT LIMITS ON LIGHT RADIONS}

Before we move on to the very important issue of the explicit nature of possible radius stabilization mechanisms as discussed in Sec. IV, it will be useful for us to examine some model-independent features of all these mechanisms. These include the existence of a light radial oscillation field $\phi$, with known couplings to standard model fields. Although such a field seems to be dangerous, we will argue below that it satisfies the various limits.

To see independent details of the stabilizing potential there is an upper bound on the mass of the radial excitation field which is useful to consider a general form for the bulk stabilizing potential $V_{\text {bulk }}(r)$. Around the equilibrium position this potential can be well approximated by the sum of just two powers of $r:^{3}$

$$
V_{\text {bulk }}(r)=M_{*}^{4}\left(A x^{a}+B x^{b}\right) .
$$

Here we have introduced the dimensionless radius variable $x \equiv r M_{*}$. In particular, following on from the discussion in Sec. II, for a stable minimum we study potentials of the form

$$
V_{\text {bulk }}(r)=M_{*}^{4}\left(\epsilon x^{\alpha}+\frac{N}{x^{\beta}}\right), \quad \alpha, \beta>0,
$$

or

$$
V_{\text {bulk }}(r)=M_{*}^{4}\left(\epsilon x^{\alpha}-\eta x^{\beta}\right), \quad \alpha>\beta>0 .
$$

As we discuss in Sec. IV the dimensionless parameter $\epsilon$ is a measure of the size of the effective bulk cosmological constant, and acts to prevent the radius from expanding to infinity. In contrast, the $N$ or $\eta$ terms prevent collapse to the UV, and arise from either inter-brane interactions, or from the kinetic energy of topologically quantized bulk gauge or scalar fields. As we will soon see, to get a large radius requires a small $\epsilon$, and/or a large $N$ or $\eta$.

\footnotetext{
${ }^{3}$ In this paper we will not explicitly consider potentials of the form $V_{\text {bulk }}(r) \sim r^{\alpha} f[\log (r)]$, for some function $f$, with only a single such term dominantly contributing to the potential energy near the equilibrium position. See Ref. [3] for a discussion of such potentials.
} 
Requiring the cancellation of the effective 4-dimensional cosmological constant at the minimum of these potentials leads to the equations

$$
\begin{gathered}
V_{\text {bulk }}^{\prime}\left(r_{0}\right)=0, \\
V_{\text {bulk }}\left(r_{0}\right)+N_{\text {wall }} \bar{f}^{4}=0 .
\end{gathered}
$$

Here we have allowed for the possibility that there is more than one wall or brane in the bulk, $N_{\text {wall }} \geqslant 1$. These provide localized sources of curvature (in principle of either sign as discussed in Sec. I A). However, for simplicity, we have assumed that all the branes have broadly similar such energy densities $f_{\text {grav }, i}^{4} \simeq \bar{f}^{4}$, and of the same sign. More general possibilities can also be analyzed.

In any case, Eqs. (31) can be used to determine $r_{0}$ and the required value of $N_{\text {wall }} \bar{f}^{4}$ in terms of the basic modeldependent parameters of the potential, $\alpha, \beta, \epsilon$, etc. Alternately, we can find the values of these parameters necessary to produce a desired internal radius $x_{0}=r_{0} M_{*}$ (in string units). Defining the useful dimensionless combination

$$
\gamma \equiv N_{\text {wall }} \frac{\bar{f}^{4}}{M_{*}^{4}}
$$

the stabilizing parameters $\epsilon$ and $N$ are determined to be

$$
\left.\begin{array}{l}
\epsilon=-\frac{\beta}{\alpha+\beta} \gamma \frac{1}{x_{0}^{\alpha}} \\
N=-\frac{\alpha}{\alpha+\beta} \gamma x_{0}^{\beta}
\end{array}\right\} \text { given } \bar{f}^{4}<0 .
$$

In the case of the potential Eq. (30),

$$
\left.\begin{array}{l}
\epsilon=\frac{\beta}{\alpha-\beta} \gamma \frac{1}{x_{0}^{\alpha}} \\
\eta=\frac{\alpha}{\alpha-\beta} \gamma \frac{1}{x_{0}^{\beta}}
\end{array}\right\} \text { given } \bar{f}^{4}>0 .
$$

Now, by equipartition, the second derivative of the general potential $V(r)_{\text {bulk }}$ of Eqs. (29) and (30) around the minimum is given by $V^{\prime \prime} \sim V\left(r_{0}\right)_{\text {bulk }} /\left(r_{0}\right)^{2}$. In addition the mean bulk value of the cosmological constant is defined by

$$
\bar{\Lambda} \equiv \frac{V\left(r_{0}\right)}{\left(r_{0}\right)^{n}} .
$$

Thus using the definition of the canonically normalized radial excitation, Eq. (26), it is easy to see that physical mass of the radial excitations is

$$
m_{\text {radial }}^{2} \sim \frac{\bar{\Lambda}}{M_{*}^{2+n}} .
$$

But now we can apply the curvature radius bound on $\bar{\Lambda}$, Eq. (4), to find

$$
m_{\text {radial }}^{2} \lesssim M_{*}^{2}\left(\frac{M_{*}}{M_{\mathrm{pl}}}\right)^{4 / n} N_{\text {wall }} \sim \frac{N_{\text {wall }}}{r_{n}^{2}}
$$

independent [up to the $\mathcal{O}(1)$ coefficients we have dropped] of any details of the stabilizing potential or mechanism. Evaluating this for the most conservative case of $N_{\text {wall }}=1$ and for the desired values of $M_{*}$ leads to a mass for the radial field that varies between $10^{-2} \mathrm{eV}$ or less for $n=2$, to $\sim 20 \mathrm{MeV}$ or less for $n=6$. Note that the reason why the radion mass is much smaller than $M_{*}$ is that $\bar{\Lambda}$ must be relatively small to allow large extra dimensions.

So, in all the models for radius stabilization that we consider, the radion field will be very light with $m_{\phi}^{2} \lesssim r_{n}^{-2}$, at most $\sim 20 \mathrm{MeV}$ for $n=6$. Thus it is necessary to study the model-independent limits on such light radions to make sure that the entire scenario is not excluded. To do this, we have to determine the coupling of radions to SM fields on our wall. At first, it seems that there is no direct coupling of the radion field to SM fields. The reason is that the couplings of SM fields to gravity all come from the induced metric on our wall, which, if the possible Nambu-Goldstone bosons on the wall are turned off so the wall is flat in the extra dimensions, depends on $g_{\mu \nu}$ but not the radion fields $g_{m n}$. However, this argument is not correct and the radion field does indeed couple to SM fields as we now show. ${ }^{4}$ Let us go to the effective theory at distances large compared to the size of the extra dimensions. The effective action is

$$
\begin{aligned}
& \int d^{4} x \sqrt{-\operatorname{det} g_{\mu \nu}}\left(-M_{\mathrm{pl}}^{2}\left\{1+\frac{n \phi}{M_{\mathrm{pl}}}+\cdots\right\} \mathcal{R}+g^{\mu \nu} \partial_{\mu} \phi \partial_{\nu} \phi\right. \\
& \left.-m_{\phi}^{2} \phi^{2}+\mathcal{L}_{S M}\left(\psi, g_{\mu \nu}\right)\right)
\end{aligned}
$$

where $\psi$ are the SM fields. Notice that since the effective 4-dimensional Planck scale depends on the size of the extra dimensions, there is $\phi$ dependence in the coefficient of $\mathcal{R}$, and that there is no explicit dependence on $\phi$ in the SM part of the Lagrangian as expected. However, there is kinetic mixing between ordinary gravity and the $\phi$ field, specifically if we expand around a flat metric $g_{\mu \nu}=\eta_{\mu \nu}+h_{\mu \nu}$, there is a mixing of the form $\phi \partial^{2} h_{\mu}^{\mu}$. Thus, even though there is no direct coupling of the SM fields to $\phi$, one is induced through this mixing. This can be seen more clearly if we first perform a Weyl rescaling to remove the $\phi$ dependence in front of the usual graviton kinetic term. The coupling to SM fields then comes from the scale-invariance violating part of the SM Lagrangian; the leading interaction is

$$
\mathcal{L}_{S M-\phi}=\frac{\phi}{M_{\mathrm{pl}}}\left(T_{S M}\right)_{\mu}^{\mu} .
$$

Note that $\phi$ interactions are suppressed for relativistic particles, while it has comparable strength to gravity for non-

\footnotetext{
${ }^{4}$ We thank Raman Sundrum and Riccardo Rattazzi for setting us straight on this point.
} 
relativistic particles. Suppose that $\phi$ is massless. As far as the long-range force between non-relativistic particles is concerned, this just amounts to a redefinition of Newton's constant $G_{N} \rightarrow G_{N \text {,non-rel. }}$, while the Newton constant governing the interaction of gravity with light $G_{N \text {,rel. }}$ retains the standard value $G_{N \text {,rel. }}=G_{N}$. However, the successful predictions for the gravitational deflection of light as well as Big-Bang Nucleosynthesis assume $G_{N \text {,non-rel. }}=G_{N \text {,rel. }}$ at least to within a few percent. Moreover, since the long range force between non-relativistic masses has been measured down to $\sim 1 \mathrm{~mm}$ without revealing any deviation from Newtonian gravity, the mass of $\phi$ must be pushed up above $\sim(1 \mathrm{~mm})^{-1}$ $\sim 10^{-3} \mathrm{eV}$. This model-independent constraint, which was also discussed in [11], is the most important limit on light radions.

We now move on to consider other possible limits on light radions coming from cosmology and astrophysics. There are two classes of worries. The first is that not only the radion but all of its Kaluza-Klein excitations can be produced in the early universe and in stars, leading to such well-known problems as the over-efficient cooling of supernovae. This concern is identical to the problem of bulk graviton overproduction which was studied in [3], and found to in some cases (namely $n=2$ extra dimensions) to constrain but not rule out our scenario. The second concerns oscillations of the radion field itself around its minimum. These may overclose the universe, and further, since these oscillations correspond to changing the size of the extra dimensions, they also lead to an oscillating 4-dimensional Newton's constant, which can be problematic. ${ }^{5}$

Therefore we now briefly discuss some aspects of the cosmology of radion fields. We will adopt here the same attitude taken in [3]. There, limits were put on the highest temperature $T_{*}$ up to which the universe could be considered "normal," that is, with the extra dimensions stabilized and energy density dominated by the radiation on our wall. Since on-the-wall interactions can produce gravitons which escape into the bulk and which in turn can variously affect the expansion rate of the universe during nucleosynthesis, overclose the universe, and unacceptably distort the background photon spectrum when they decay, the normalcy temperature $T_{*}$ was limited to $\sim$ few $\mathrm{MeV}$ to $\sim 1 \mathrm{GeV}$ for $n=2-6$. Fortunately, $T_{*} \geq 1 \mathrm{MeV}$ in all cases (with $n=2$ marginal), so that the successful predictions of primordial nucleosynthesis can still hold in our scenario. In our present analysis of radion cosmology, we will be content to show the cosmological safety of the scenario at temperatures $\lesssim T_{*}$. Namely, we will assume that the early universe at temperatures $\gtrsim T_{*}$ evolved into a state with the radion stabilized, with the energy density stored in radion oscillations small enough to never overclose the universe. We will show that this is enough to guarantee negligible variations in $G_{N}$, and that

\footnotetext{
${ }^{5} \mathrm{We}$ remark that high-frequency oscillations of $G_{N}$ of sufficiently small amplitude around a mean value equal to the standard value of $G_{N}$ can be accommodated, despite the fact that in such a case $d G_{N} / d t$ can be significantly larger than the usually quoted bounds.
}

subsequent interactions with SM fields on the wall will not significantly excite the radion away from its minimum. ${ }^{6}$

First, note that since $T_{*} \ll \mathrm{TeV}$, the Hubble expansion rate at all times of interest satisfies $H \sim T^{2} / M_{\mathrm{pl}} \ll(1 \mathrm{~mm})^{-1}$, so that the expansionary "friction" term cannot stop $\phi$ from oscillating. Further, since $\phi$ is so light and gravitationally coupled, it is essentially stable cosmologically. The energy density $\rho_{\phi}$ stored in its oscillations redshifts away as $1 / R^{3}$, so that $\rho / T^{3}$ is invariant. It is easy to see that in order for $\phi$ to never dominate the energy density of the universe, we must have

$$
\frac{\rho_{\phi}}{T^{3}} \lesssim \frac{\rho_{\text {crit. now }}}{T_{\text {now }}^{3}} \sim 3 \times 10^{-9} \mathrm{GeV} .
$$

Using $\rho_{\phi} \sim m_{\phi}^{2} \phi^{2}$, this is enough to show that the variations in $G_{N}$ are miniscule at all epochs $T \leqslant T_{*}$ :

$$
\frac{\delta G_{N}}{G_{N}}=n \frac{\delta r}{r_{0}} \sim \frac{\phi}{M_{\mathrm{pl}}} \sim \frac{\sqrt{\rho_{\phi}}}{m_{\phi} M_{\mathrm{pl}}} \lesssim 10^{-12} .
$$

Furthermore, interactions with the SM fields cannot significantly excite the radion into oscillation. Note that it is only the excitations of $\phi$ [and not its associated Kramers-Kronig (KK) modes] which would correspond to changing the radius of the extra dimensions (and hence varying $G_{N}$ ) on cosmological scales. This single mode has couplings suppressed by the ordinary 4-dimensional Planck scale $M_{\mathrm{pl}}$, and it is therefore very difficult to excite. Quantitatively, the rate at which collisions of SM particles dump energy into $\phi$ is

$$
\dot{\rho}_{\phi} \sim \frac{T^{7}}{M_{\mathrm{pl}}^{2}} .
$$

The total amount of energy dumped into $\phi$ during a Hubble time is then

$$
\delta \rho_{\phi} \sim \frac{T^{5}}{M_{\mathrm{pl}}},
$$

leading to an unobservably small variation in $G_{N}$

$$
\frac{\delta G_{N}}{G_{N}} \sim \frac{\sqrt{\delta \rho_{\phi}}}{m_{\phi} M_{\mathrm{pl}}} \sim\left(\frac{T^{5}}{(1 \mathrm{TeV})^{4} M_{\mathrm{pl}}}\right)^{1 / 2} \lesssim 10^{-18} .
$$

Of course, this is hardly surprising. Recall that at temperatures below $T_{*}$, the energy dumped into the bulk gravitons and $\phi$ together with all of their KK excitations never overclose the universe. Even if all of this energy was somehow transferred into moving the single mode $\phi$, we already found that as long as the $\phi$ energy did not overclose the universe, the variations in $G_{N}$ are negligible.

\footnotetext{
${ }^{6} \mathrm{~A}$ full discussion of the very early universe cosmology in our scenario, in particular, the worry that an early period of inflation could lead to a form of the Polonyi problem involving $\phi$ will appear in Ref. [16].
} 
Similar comments apply to radion excitation in stars: the energy lost to the production of $\phi$ together with all its $\mathrm{KK}$ excitations are safe for the same reason as bulk graviton production is safe (see Ref. [3]), while the single mode $\phi$ is too weakly coupled to be perturbed enough for significant variations of $G_{N}$ to be observable. For instance, in the collapse of SN 1987A over a time $t_{\mathrm{SN}} \sim 1 \mathrm{~s}$, the variation in $G_{N}$ can be estimated as above, yielding

$$
\left.\frac{\delta G_{N}}{G_{N}}\right|_{\mathrm{SN}}=n \frac{\delta r}{r_{0}} \sim\left(\frac{T_{\mathrm{SN}}^{n+7} t_{S N}}{M_{*}^{n+2} m^{2} M_{\mathrm{pl}}^{2}}\right)^{1 / 2} \ll 1,
$$

even in the worst case $T_{\mathrm{SN}} \sim 100 \mathrm{MeV}, n=2$. Thus the local variation of $G_{N}$ is harmless for any number of extra dimensions $n=2-6$.

\section{RADIUS STABILIZATION MECHANISMS}

We now turn to some explicit mechanisms by which internal dimensions may be stabilized at a radius much greater than the fundamental Planck length $\sim M_{*}^{-1}$. Two issues must be distinguished in discussing radius stabilization: the mechanism by which the internal dimensions are prevented from collapsing to $1 / M_{*}$, and the mechanism by which they are prevented from expanding to a size much larger than a millimeter or Fermi.

\section{A. Generalities}

The most obvious idea for limiting the expansion of the internal dimensions is to employ a component of the potential energy that scales like the volume of the internal space: $V \sim r^{n}$. Such an effective potential energy density results from a positive bulk cosmological constant $\Lambda$, which gives $V(r) \sim \Lambda r^{n}$ as shown in Sec. II. As we have already discussed the size of this bulk cosmological constant must be small. However, while we have no compelling explanation for the size of this bulk cosmological constant, it is interesting that its smallness can at least be stable under radiative corrections. Suppose that the short-distance theory of gravity (perhaps string theory) is supersymmetric, with the supersymmetries broken only on the walls at a scale $\sim M_{*} \sim|\bar{f}|$ $\sim 1 \mathrm{TeV}$. It is easy to see that the Bose-Fermi splittings induced in the bulk supergravity multiplet are then [2]

$$
\left|m_{\text {bose }}^{2}-m_{\text {fermi }}^{2}\right|_{\text {bulk }} \sim N_{\text {wall }} \frac{M_{*}^{4}}{M_{\mathrm{pl}}^{2}}
$$

so that the quantum corrections to the bulk cosmological constant would be of order

$$
\left|\Lambda_{\text {quant }}\right| \sim\left|m_{\text {bose }}^{2}-m_{\text {fermil bulk }}^{2} M_{*}^{(2+n)} \sim\right| \Lambda_{\text {tree }} \mid,
$$

and the small value of the cosmological constant can be technically natural.

We now turn to the ways in which the radii of the extra dimensions can be stopped from collapsing to small values. We will see that a wide range of mechanisms are in principle possible, leading to a variety of power-law potentials of the form $1 / r^{l}$ for various $l$. One minimal possibility is if the compact manifold has (positive) curvature, in which case

$$
V_{\text {bulk }}(r) \sim \Lambda r^{n}-M_{*}^{n+2} r^{n-2} .
$$

As can readily be seen from Eq. (34), this will require a large positive value for the ratio $\gamma=N_{\text {wall }} \bar{f}^{4} / M_{*}^{4}$, which can arise if we have a configuration of a large number $N_{\text {wall }} \gg 1$ of branes. This possible "brane lattice crystallization," together with various generalizations, will be discussed in the next subsection. Alternately, if we wish to compactify on manifolds with no curvature (tori), the ultraviolet stabilization can be provided by dynamics conserving a topological number $k$, which we will explore in Sec. IV C.

\section{B. Radius stabilization from brane lattice crystallization}

The largeness of the internal dimensions compared to $(1 \mathrm{TeV})^{-1}$ can arise from the existence of a large (conserved) number of branes populating the bulk. There can exist inter-brane forces which act like the Van der Walls and hard-core forces between atoms in a crystal. The inter-brane distance is set by these forces, and might be quite small, but the size of the whole internal space is set by the total number of branes, just as the total extent of a crystal is set by the number of atoms, rather than just the inter-atom distance which is much smaller.

\section{Minimal scenario}

We will motivate stabilizing the extra dimensions with large brane numbers by considering the minimal example of compact manifolds with positive curvature, which together with a positive bulk cosmological constant give a bulk potential of the form

$$
V_{\mathrm{bulk}}=\Lambda r^{n}-M_{*}^{n+2} r^{n-2} \text {. }
$$

For $n=2$, the curvature contribution to the potential is constant and does not play any role in radius stabilization (although it does contribute an extra term to the effective 4-d cosmological constant). For $n>2$, however, this potential has a stable minimum. From Eq. (34), we find that a large value for $\gamma$ is needed, $\gamma \sim\left(M_{*} r_{0}\right)^{n-2}$. For simplicity, we will assume that all the branes are broadly similar with $\bar{f}^{4}$ $\sim M_{*}^{4}$. Then, we must have a large number of branes

$$
N_{\text {wall }} \sim\left(M_{*} r_{0}\right)^{n-2} \sim\left(\frac{M_{\mathrm{pl}}}{M_{*}}\right)^{2(n-2) / n} .
$$

Numerically this varies from $N_{\text {wall }} \sim 10^{10}$ for $n=3$ to $N_{\text {wall }}$ $\sim 10^{20}$ for $n=6$.

Although this is a large number it is not so large as to lead to problems. Specifically, we note that there is a constraint on the total number of branes that can populate the internal dimensions. If the transverse inter-brane separation becomes comparable to $1 / M_{*}$, then there will be new light open string modes that arise from strings starting on one brane and ending on a neighbor. Thus the maximum number of branes that can occupy the extra dimensions is 


$$
N_{\text {wall, } \max } \sim\left(r_{0}\right)^{n} M_{*}^{n} \sim\left(\frac{M_{\mathrm{pl}}}{M_{*}}\right)^{2} \sim 10^{32},
$$

which is considerably greater than the necessary number Eq. (50).

However, with such a large number of branes, it is obviously important to ensure that some dynamics forces them to spread out in the bulk and not sit on top of each other. This can easily be arranged. We know that there is a gravitational force between the branes, and if they carry any sort of (like sign) gauge charge there will also be an opposite gauge force between them with exactly the same dependence on interbrane separation. In fact, when the charge density $\rho$ is equal to the tension $T$, there can be an exact cancellation of the inter-brane forces. This is what happens in the case of supersymmetric D-branes. Polchinski's now classic calculation of the forces between D-branes demonstrated that the forces due to Ramond-Ramond gauge fields precisely canceled the gravitational forces in the supersymmetric limit, as they must for a pair of BPS states, which satisfy $T=\rho$. If there is a mismatch between the charge and tension of the branes, the net force between a pair of branes can be made repulsive, forcing them to spread out uniformly in the bulk. Of course, we must now take the inter-brane potential energy into account in the energetics, but interestingly, this effect is parametrically of the same order as the terms in the potential we already have. By Gauss' law, the potential between branes falls off with the inter-brane separation $r$ according to the Coulomb potential in the transverse $n$ dimensions $V_{\text {int. }}(r)$ $\sim \rho^{2} / r^{n-2}$.

If we first imagine just two 3-branes populating the internal space the potential energy varies as

$$
V(r) \sim M_{*}^{4} \frac{1}{\left(r M_{*}\right)^{n-2}} .
$$

Here we have taken the effective net charge density on the wall to be $M_{*}^{4}$, as we would expect if supersymmetry is broken at a scale $|f| \sim M_{*}$. The inter-brane distance can be estimated from balancing this repulsive force against a bulk cosmological constant term $V(r) \sim \bar{\Lambda} r^{n}$. Imposing the cancellation of the 4-dimensional cosmological constant Eq. (5) leads to an inter-brane separation $r_{I}$

$$
\left(r_{I}\right)^{n-2} \sim \frac{M_{*}^{4}}{M_{*}^{n+2}} \sim(1 \mathrm{TeV})^{(2-n)} .
$$

What happens when $N_{\text {wall }}$ branes occupy the internal space? One may think that the size of the internal volume will just be $N_{\text {wall }}$ times larger than $\left(r_{I}\right)^{n}$ calculated above. However this is incorrect. The reasons for this are two-fold. The first is that, unlike in a normal crystal, there is no necessity that the inter-brane forces are screened. Thus the total potential energy density due to the inter-brane forces increases as $N_{\text {wall }}^{2}$, just as the gravitational potential in a star, and the UV stabilizing part of the potential has the form

$$
V \sim M_{*}^{4} N_{\text {wall }}^{2} \frac{1}{\left(r M_{*}\right)^{n-2}},
$$

where $r$ is now roughly the total extent of the system. The second reason why the two brane calculation is modified is that the equation for the cancellation of the effective 4-dimensional IR cosmological constant is modified to Eq. (10). Putting all parts of the potential together, we have

$$
V(r)_{\mathrm{tot}} \sim M_{*}^{4} N_{\text {wall }}^{2} \frac{1}{\left(r M_{*}\right)^{n-2}}+\bar{\Lambda} r^{n}+N_{\text {wall }} f^{4}
$$

Solving for the size of the system gives,

$$
r_{0} \sim\left(\frac{N_{\text {wall }}^{2}}{\bar{\Lambda} M_{*}^{n-6}}\right)^{1 / 2(n-1)} .
$$

However $\bar{\Lambda}$ can be eliminated by imposing Eq. (10), with $|f| \sim M_{*}$ leading to

$$
\bar{\Lambda} \sim \frac{M_{*}^{(n+4)}}{N_{\text {wall }}^{2 /(n-2)}}
$$

and thus the final expression for $r_{0}$

$$
r_{0} \sim \frac{N_{\text {wall }}^{1 /(n-2)}}{M_{*}} .
$$

Utilizing the formula for the required size of the extra dimensions, $\left(r_{0}\right)^{n} M_{*}^{n+2}=M_{\mathrm{pl}}^{2}$, we can solve for the necessary brane-number

$$
N_{\text {wall }} \sim\left(\frac{M_{\mathrm{pl}}}{M_{*}}\right)^{2(n-2) / n}
$$

exactly the expression Eq. (50).

Notice that if one substitutes this value back into the equation for $\bar{\Lambda}$, Eq. (57), then one finds

$$
\bar{\Lambda} \sim M_{*}^{n+4}\left(\frac{M_{*}}{M_{\mathrm{pl}}}\right)^{4 / n},
$$

which is smaller than the naive value $M_{*}^{n+4}$, showing that indeed one component of the hierarchy problem in this framework is the (bulk) cosmological constant problem. As discussed above, there is one other requirement that needs to be satisfied. The mean curvature radius on scales smaller than the inter-brane separation needs to be larger than the inter-brane separation itself. The average inter-brane transverse separation is now

$$
r_{I} \equiv\left(\frac{\left(r_{0}\right)^{n}}{N_{\text {wall }}}\right)^{1 / n} \sim \frac{1}{M_{*}}\left(\frac{M_{\mathrm{pl}}}{M_{*}}\right)^{4 / n^{2}}
$$

whilst the curvature radius resulting from our potential is 


$$
L_{\mathrm{curv}} \sim \frac{1}{M_{*}}\left(\frac{M_{\mathrm{pl}}}{M_{*}}\right)^{2 / n} .
$$

For the case of $n>2$ where the above analysis applies, one always has $L_{\text {curv }}>r_{I}$ as required.

In addition if the supersymmetries of string theory are broken only by on-the-wall dynamics at a scale $\sim M_{*}$ $\sim 1 \mathrm{TeV}$, then the mass splittings so induced among the bulk supergravity multiplet are $\sim M_{*}^{2} / M_{\mathrm{pl}} \sqrt{N_{\text {wall }}}$, and a bulk cosmological constant of order $\Lambda \sim\left(M_{*}^{2} / M_{\mathrm{pl}} \sqrt{N_{\text {wall }}}\right)^{(4+n)}$ arises. Then the ratio of this new term to the $\bar{\Lambda}$ term is

$$
\frac{\Lambda_{\text {quant. }}}{\Lambda_{\text {tree }}} \sim\left(\frac{M_{*}}{M_{\text {pl }}}\right)^{(2 n+4) / n} \ll 1 .
$$

Therefore the value of the bulk cosmological constant can still be technically natural in the case of a large number, $N_{\text {wall }}$, of branes.

In summary, we have made a number of simplifying assumptions which can be questioned and modified. These include the simplification that all 3-branes are broadly similar and have tensions and charge densities $\sim(1 \mathrm{TeV})^{4}$ with a mismatch that is also of order $(1 \mathrm{TeV})^{4}$. Nevertheless, it is encouraging that the large-brane-number scenario for stabilizing the volume of the internal dimensions at large values passes the first tests.

\section{Non-extensive bulk cosmological constant}

There is another interesting possibility where the size of the extra dimensions is completely explained by a large brane number without needing to invoke another small parameter (the small bulk cosmological constant in the above analysis). Suppose that the IR potential is $\lambda r^{a}$, for $a<n$ with the normal $\Lambda r^{n}$ term being sub-dominant. The bulk potential then reads

$$
V(r)_{\mathrm{bulk}} \sim \frac{N_{\mathrm{wall}}^{2}}{M_{*}^{n-6} r^{n-2}}+\lambda r^{a} .
$$

With this potential we still need the same large brane number $N_{\text {wall }} \sim\left(M_{*} r_{0}\right)^{n-2}$. The size of $\lambda$ is to be

$$
\lambda \sim M_{*}^{4+a}\left(\frac{M_{*}}{M_{\mathrm{pl}}}\right)^{2(a+2-n) / n} .
$$

For $a=n-2$, the required value for $\lambda$ agrees with the natural value $\sim M_{*}^{4+a}$. This is intriguing, since $a=n-2$ is precisely the power associated with curvature terms. Of course, a compact manifold with positive curvature makes the wrong-sign contribution to the potential, but we can choose the compact manifold to have negative curvature. For instance, genus $g$ $>1$ Riemann surfaces have negative Euler characteristic and hence negative average curvature by the Gauss-Bonnet theorem. In order to stabilize more than two dimensions in this way, we can compactify on direct products of such Riemann surfaces, which will then give the correct exponent and the correct sign in the potential of Eq. (64).

\section{Casimir forces between branes $^{7}$}

Another potentially attractive idea for UV stabilization at the quantum level is to use the Casimir force to maintain the size of the internal space [14]. The effective $4 d$ potential energy density corresponding to the Casimir effect in a (4 $+n$ )-dimensional spacetime is

$$
V(r) \sim \frac{C}{r^{4}}
$$

where $C$ is a calculable coefficient in any given model. Even with a general non-extensive stabilizing potential, $\Delta V \sim \lambda r^{a}$ this leads to a inter-brane distance of

$$
r_{I} \sim\left(\frac{C}{\lambda}\right)^{1 /(4+a)}
$$

Given that the "natural" value of $\lambda$ is expected to be $M_{*}^{(4+a)}$, this clearly does not allow us to stabilize at large radii. What about many branes? The problem is that, when we go to $N_{\text {wall }} 3$-branes in the bulk, the Casimir energy does not increase with $N_{\text {wall }}$ for $n \geqslant 2$. But the total wall cosmological constant $N_{\text {wall }} \bar{f}^{4}$ does, and thus the situation gets worse.

In summary, the Casimir force idea, even with a large brane number $N_{\text {wall }} \gg 1$, fails to stabilize the internal dimensions at large radii, at least under the simplifying assumptions we have made.

\section{Topological stabilization}

One of the most attractive ways of preventing collapse is to imagine that there is a topologically conserved quantity which holds up the size of the extra dimensions. A prototypical example of this is provided by the monopole stabilization mechanisms discussed in Ref. [15] and in the context of our scheme by Sundrum [11]. Consider the simple case of two extra dimensions and where the internal manifold has the topology of a 2-sphere, $S^{2}$. Further suppose that in the bulk there exists not only the graviton, but also a U(1) gauge field, which might naturally be a Ramond-Ramond (RR) gauge field of the string theory in question. Then it is possible to take the gauge field configuration on $S^{2}$ to be topologically non-trivial with quantized "monopole number'" $k$ [the first Chern number of the $\mathrm{U}(1)$ bundle] given by ${ }^{8}$

$$
\frac{1}{2 \pi} \int_{S^{2}} H^{(2)}=k \text {. }
$$

If the area of the $S^{2}$ is denoted $V_{(2)}$ then we have $H$ $\sim k / V_{(2)}$ and since the kinetic term for the U(1) gauge field is (expressed in form notation, with $M^{4}$ denoting 4-dimensional Minkowski space)

\footnotetext{
${ }^{7}$ This possibility was also analyzed by Sundrum [11].

${ }^{8}$ We will always use $H$ for field strengths of gauge fields that live in the bulk. Quite often we will think of these as being RR gauge fields.
} 


$$
S_{K E} \sim \frac{M_{*}^{2}}{g^{2}} \int_{M^{4} \times S^{2}} H \wedge * H,
$$

we have that the 4-dimensional potential energy density of the monopole field on the $S^{2}$ scales like

$$
V \sim \frac{M_{*}^{2}}{g^{2}} \frac{k^{2}}{V_{(2)}}
$$

In other words we get an energy density that scales like $k^{2} M_{*}^{2} /\left(g^{2} r^{2}\right)$. For large enough monopole number, $k$, this will stabilize the internal $S^{2}$ at any desired size.

This basic mechanism has a wide variety of generalizations. One such is to use the topological invariants of the higher-form RR gauge fields that naturally arise in the type II and type I string theories with D-branes. Let us recall here that for stabilizing $n>2$ dimensions topologically, we must work with compact manifolds of zero curvature i.e. tori, since otherwise the curvature term will dominate the dynamics and we must revert to the analysis of the previous subsection.

\section{Higher-form RR fields}

Denote the manifold of the extra $n$ dimensions by $E^{n}$, and suppose that the bulk theory contains an $(n-1)$-form $\mathrm{U}(1)$ gauge field, with $n$-form field strength $F^{(n)}$. Then once again there is the topological invariant

$$
\frac{M_{*}^{n-2}}{2 \pi} \int_{E^{n}} H^{(n)}=k .
$$

The kinetic energy of $H^{(n)}$ is the generalization of the usual 1-form gauge kinetic term

$$
S_{K E, n} \sim \frac{M_{*}^{n}}{g^{2}} \int_{M^{4} \times E^{n}} H^{(n)} \wedge * H^{(n)},
$$

and thus the potential energy density depends on the volume $V_{(n)}$ of $E^{n}$ as

$$
V \sim \frac{1}{g^{2} M_{*}^{n-4}} \frac{k^{2}}{V_{(n)}} .
$$

In the case of the chiral type IIB string theory there exists 1 and 3-form RR field strengths and a self dual 5-form RR field strength (together, of course, with their magnetic duals). There also exists the usual Neveu-Schwarz-Neveu-Schwarz (NS-NS) 3-form field strength. The type I string theory has a 3-form RR field strength and it's 7-form magnetic dual. Thus using the invariants so far described, it is natural to stabilize 1,3 , and 5-manifolds.

However, invariants that lead to $1 / r^{n}$ potentials for $E^{n}$ are not the only possibility. Consider the situation in which our 3-brane world is the boundary of (a set of) higherdimensional branes which are in turn embedded in the full $(4+n)$-dimensional space. We can then use topological invariants of the world-volume gauge fields of these higher- dimensional branes to stabilize the internal dimensions. To make this clear consider the following very simple example: In the $n=4$ case take the internal manifold to be $E^{4}=T_{1}^{2}$ $\times T_{2}^{2}$. Suppose further that there exist 25 -branes that intersect at the position of our 3-brane but are perpendicular in the extra 4 dimensions, so that one 5-brane lives in $M^{4}$ $\times T_{1}^{2}$ and the second lives in $M^{4} \times T_{2}^{2}$. Then we have the two topological invariants

$$
\frac{1}{2 \pi} \int_{T_{i}^{2}} F_{i}=k_{i}, \quad i=1,2,
$$

where $F_{i}, \quad i=1,2$ are world-volume U(1) 2-form field strengths of the first and second 5-brane. The brane-localized kinetic terms for these gauge fields then leads to an effective 4-dimensional potential energy density of the form

$$
\Delta V(r) \sim \frac{M_{*}^{2} k_{1}^{2}}{r_{1}^{2}}+\frac{M_{*}^{2} k_{2}^{2}}{r_{2}^{2}},
$$

where $r_{1}$ and $r_{2}$ are the radii of the two $T^{2}$ 's. Note that since we have used tori, there is no negative curvature term $\sim-M_{*}^{2} r^{2}$ in the potential. This, then, is an UV stabilizing potential for $E^{4}=T_{1}^{2} \times T_{2}^{2}$ not of the form $1 / r^{4}$. Clearly this type of mechanism admits many generalizations.

Finally, one can also consider higher "reducible" invariants such as the second Chern class of the usual 2-form U(1) field strength defined with respect to a 4-manifold

$$
\frac{1}{8 \pi^{2}} \int_{E^{4}} H^{(2)} \wedge H^{(2)}=c_{2},
$$

but such invariants typically lead to a potential energy varying as $r^{\alpha}$ with $\alpha \geqslant 0$.

\section{Metric topological invariants}

Purely metric topological invariants are possible, for example the Euler number of a 2-manifold component $E^{2}$ of the internal space

$$
\chi=\frac{1}{2 \pi} \int_{E^{2}} R
$$

where $R$ is the curvature 2 -form. Other possibilities include the Pontrjagin classes of the tangent bundle of the internal manifold. However, because the leading term in the gravitational effective action is only linear in the curvature, this does not provide a UV stabilizing potential unless higher derivative terms, such as

$$
\Delta S \sim M_{*}^{n} \int \operatorname{tr}\left(R^{2}\right)
$$

are included in the effective action. For the simple case of $n=2$ this leads to a potential $V \sim \chi^{2} M_{*}^{2} / r^{2}$. For this to balance, at the appropriate $r_{0}$, even a best-case stabilizing potential of the form $M_{*}^{5} r$, an Euler number of $\chi$ $\sim\left(M_{\mathrm{pl}} / M_{*}\right)^{3 / 2}$ is required. So clearly the internal manifold 
is very highly curved. In particular, the leading gravitational action $M_{*}^{4} \int \mathcal{R}$ dominates the other terms by an amount $\left(M_{\mathrm{pl}} / M_{*}\right)^{1 / 2}$, and leads to an unacceptably large bulk cosmological constant. This seems to be a generic problem with this type of topological stabilization, although we have not investigated the question in detail.

\section{Scalar-field and other non-gauge invariants ${ }^{9}$}

One can also imagine stabilizing the size of the internal space by the use of non-gauge or metric topological invariants. For example, consider a complex scalar field that lives on a 1-dimensional higher brane that has as boundary our 3-brane. Then the phase of this field can wind as an $S^{1}$ cycle of the internal space is transversed, with topologically conserved winding number

$$
k=\int_{S^{1}} d \phi
$$

Once again the kinetic energy of this configuration increases as the size of the internal space is reduced, and thus a stabilizing potential results. In order to stabilize more than one dimension in this way, we can have $n$ different scalar fields living on $n$ different 4-branes which have the 3 dimensions of our 3-brane in common but have mutually orthogonal fourth spatial dimensions. The $i$ th scalar field can wrap around the 4th dimension of the $i$ th 4-brane, generating a potential of the form $r_{1}^{-1}+\cdots r_{n}^{-1}$. Together with a bulk cosmological constant giving a potential $\sim\left(r_{1} \cdots r_{n}\right)$, this can stabilize all the $n$ dimensions. More sophisticated scalar field invariants are also conceivable, the Hopf winding number of the map $\pi: S^{3} \rightarrow S^{2}$ being one among many such examples. In general scalar field invariants lead to quite similar results to the gauge field topological stabilization mechanisms, but possibly without the natural advantage of gauge fields of their constrained couplings. (For instance it is easy to arrange that the stabilizing gauge fields do not lead to dangerous flavor-changing neutral current processes on the wall, while this requires additional input in the scalar case.)

\section{Phenomenologically successful topological stabilization}

In the previous subsections we have seen that a variety of UV stabilizing potential energy densities of the general form $M_{*}^{4}\left[k^{2} /\left(r M_{*}\right)^{\beta}\right]$ are possible. We now wish to get an idea of the numerical values the various parameters must take to stabilize the radii at the desired sizes. From Eq. (33), we have that

$$
k^{2} \sim \frac{\left|N_{\mathrm{wall}} \bar{f}^{4}\right|}{M_{*}^{4}}\left(M_{*} r_{0}\right)^{\beta} \gtrsim\left(\frac{M_{\mathrm{pl}}}{M_{*}}\right)^{2 \beta / n}\left(\frac{1 \mathrm{TeV}}{M_{*}}\right)^{4}
$$

where the inequality comes from imposing the constraint that the radion is heavier than $\sim(1 \mathrm{~mm})^{-1}$. In the above examples of topological radius stabilization the quantity $k$ is

${ }^{9}$ Gia Dvali has independently considered this possibility. We thank him for discussions. directly proportional to the "monopole" number. From this expression the smallest $k$ clearly occurs when the ratio $\beta / n$ is as small as possible. As an example, if $\beta=1$ and there are 6 extra dimensions, then for $M_{*} \sim 1 \mathrm{TeV}, k \gtrsim 3 \times 10^{2}$ is required to stabilize at a sufficiently large radius. If $\beta=2$ and there are 6 extra dimensions, then $k \gtrsim 10^{5}$ is necessary. The $\beta=2$ case is particularly interesting since it is the first case we can realize with gauge-field topological invariants rather than scalar field invariants. The worst case, requiring the largest $k$, occurs when $\beta / n$ takes on its largest value. A typical "worst-case"' is provided by the irreducible topological stabilization mechanism involving bulk RR fields (for example). This gives $\beta=n$, and leads to $k \gtrsim 10^{15}$.

Note that in the special case with $\beta=1$ and $n=6$, if we are willing to move the fundamental scale $M_{*}$ up to $\sim 10 \mathrm{TeV}$, while keeping $N_{\text {wall }} \bar{f}^{4} \sim(1 \mathrm{TeV})^{4}$, we can get away with $k \sim 1$. This may not be unnatural, after all, one could easily imagine that the scale $\bar{f}$ is $\sim 10$ smaller than the scale $M_{*}$ due to partial cancellations up to some 1 or 2-loop order. Also recall that as shown in Sec. IV A, the small value of the bulk cosmological constant is unexplained but is at least technically natural, if SUSY is primordially broken on the walls.

In summary we have shown that the topological stabilization mechanism successfully meets all our phenomenological requirements, with a price of a large, but in some cases not too large integer $k$.

\section{Corrections to leading-order potentials}

Finally, one may worry that in the regime of interest, when $r \sim r_{0}$, the semiclassical reasoning that we have applied to the leading-order kinetic and non-derivative terms in the effective action suffers from large corrections due to the presence of other terms. Such corrections are, in actual fact, entirely negligible. For example, if one included higher-order derivative terms, such as

$$
\Delta S \sim M_{*}^{n-4} \int_{M^{4} \times E^{n}}\left(H^{(p)} \wedge H^{(p)}\right) \wedge *\left(H^{(p)} \wedge H^{(p)}\right),
$$

in the effective action, then they would lead to corrections in the 4-dimensional effective potential energy density, $V$, of order

$$
\Delta V \sim M_{*}^{4} \frac{k^{4}}{\left(M_{*} r_{0}\right)^{3 n}}
$$

at the minimum $r_{0}$. Compared to the leading kinetic term this is a fractional change of order

$$
\frac{\Delta V}{V} \sim k^{2}\left(\frac{M_{*}}{M_{\mathrm{pl}}}\right)^{4}
$$

negligible unless $k \gtrsim 10^{30}$. Such statements generally apply for $r \sim r_{0}$, and are basically due to the fact that $r_{0}$ $\gg(1 \mathrm{TeV})^{-1}$. This is not quite trivial because of the potentially large dimensionless factor $k$ which could have over- 
come this suppression. In any case we see that the leadingorder analysis is entirely sufficient unless we are interested in physics at radii $r \ll r_{0}$.

\section{REMARKS AND SUMMARY}

The hierarchy problem in our framework is replaced by the problem of obtaining large new dimensions, of a size which varies between a millimeter and a fermi depending on the number of new dimensions, in a theory with a much smaller fundamental length $\sim \mathrm{TeV}^{-1}$. In this paper we exhibited mechanisms which provide such large extra dimensions. These mechanisms relied on two ingredients.

A large conserved integer $Q$, which can be a large number $N_{\text {wall }}$ of branes, or the topological charge $k$ of the vacuum configuration. This large integer should be regarded as analogous to the net conserved baryon number which accounts for the large size of macroscopic objects relative to that of atoms. The necessity for such a large number was not forced on us by the need for large internal dimensions, but rather by the requirement that the radial oscillation field (or "radion") be sufficiently heavy to have escaped tests of gravity at the millimeter-scale and above. The value of $Q$ depends on the details of the stabilization scenario; it varies from $N_{\text {wall }}$ $\sim 10^{10}$ to $N_{\text {wall }} \sim 10^{20}$ in the brane-lattice-crystallization scenario, while in the topological stabilization scenario it varies from $k \sim 1$ to $k \sim 10^{15}$.

A small bulk cosmological constant, analogous to the 4-dimensional cosmological constant whose smallness accounts for the size of our universe relative to the Planck length. However, as we discuss in detail in, for example, Sec. IV A, the value of this bulk cosmological constant is stable against radiative corrections if supersymmetry-breaking of order of the fundamental Planck mass $\sim M_{*} \sim 1 \mathrm{TeV}$ takes place on the 3-branes. Of course we must still impose a fine tuning to get a vanishing effective 4-dimensional, branelocalized cosmological constant in the IR in our world. This is expressed in Eq. (5) or (10), depending on the stabilization scenario.

A valid criticism of our analysis is that we have not pro- vided a dynamical framework in which, for instance, the largeness of $Q$ or $k$ is explained. As discussed in the Introduction our viewpoint on this issue is that this is closely analogous to the situation in the MSSM where soft supersymmetry-breaking operators of order $(1 \mathrm{TeV})$ are introduced [13].

With the advent of many quantum-field-theoretic (QFT) models of dynamical supersymmetry breaking it is commonly believed that the problem of the size of these soft operators has been solved, at least in principle. However, from a fundamental vantage-point this belief is not correct. Concretely, what is the situation in the standard model or MSSM, where the usual (reduced) Planck mass $M_{\mathrm{pl}} \sim 2$ $\times 10^{18} \mathrm{GeV}$ is taken as fundamental? We must now explain the ratio of this Planck scale to the weak scale $\sim 10^{15}$. There too the "dilaton runaway problem" prevents us from having a calculational framework for this number. This point is important to emphasize. Although in the context of QFT dynamical SUSY breaking solves the hierarchy problem, in that it generates the small scale by dimensional transmutation, in the context of string theory the couplings and thus the scale of SUSY breaking are dynamical, and there is a ground state at zero coupling with unbroken supersymmetry [17]. This means that there exists no known solution to the hierarchy problem in usual 4-dimensional QFT once it is embedded in string theory. Therefore both frameworks face similar challenges.

\section{ACKNOWLEDGMENTS}

It is a pleasure to thank Philip Argyres, Gia Dvali, Michael Graesser, Vadim Kaplunovsky, Yaron Oz, Riccardo Rattatzi, Eva Silverstein, and Raman Sundrum for valuable discussions. S.D. thanks the CERN theory group for its hospitality. The work of N.A.H. was supported by the Department of Energy under contract DE-AC03-76SF00515. The work of S.D. was supported in part by NSF grant PHY9219345-004. The work of J.M.R. was supported in part by the A.P. Sloan Foundation.
[1] N. Arkani-Hamed, S. Dimopoulos, and G. Dvali, Phys. Lett. B 429, 263 (1998).

[2] I. Antoniadis, N. Arkani-Hamed, S. Dimopoulos, and G. Dvali, Phys. Lett. B 436, 257 (1998).

[3] N. Arkani-Hamed, S. Dimopoulos, and G. Dvali, Phys. Rev. D 59, 086004 (1999).

[4] See, for example, J. Polchinski, "TASI lectures on D-branes,", hep-th/9611050; C. Bachas, "Lectures on D-branes,", hep-th/9806199.

[5] K.R. Dienes, E. Dudas, and T. Gherghetta, Phys. Lett. B 436, 55 (1998); Nucl. Phys. B537, 47 (1999); K.R. Dienes, E. Dudas, T. Gherghetta, and A. Riotto, ibid. B543, 387 (1999).

[6] I. Antoniadis, Phys. Lett. B 246, 377 (1990).

[7] P. Horava and E. Witten, Nucl. Phys. B460, 506 (1996); E. Witten and J.D. Lykken, Phys. Rev. D 54, 3693 (1996); E.
Caceres, V.S. Kaplunovsky, and I.M. Mandelberg, Nucl. Phys. B493, 73 (1997).

[8] G. Shiu and S.H.H. Tye, Phys. Rev. D 58, 106007 (1998).

[9] C. Bachas, J. High Energy Phys. 11, 023 (1998).

[10] R. Sundrum, Phys. Rev. D 59, 085009 (1999).

[11] R. Sundrum, Phys. Rev. D 59, 085010 (1999).

[12] P. Argyres, S. Dimopoulos, and J. March-Russell, Phys. Lett. B 441, 96 (1998).

[13] S. Dimopoulos and H. Georgi, Nucl. Phys. B193, 150 (1981).

[14] P. Candelas and S. Weinberg, Nucl. Phys. B237, 397 (1984).

[15] Y. Okada, Phys. Lett. 150B, 103 (1985).

[16] N. Arkani-Hamed, S. Dimopoulos, and J. March-Russell (in preparation).

[17] M. Dine and N. Seiberg, Phys. Lett. 162B, 299 (1985). 